\title{
Expression and subcellular localization of pre-rRNA processing factor homologues in higher plants
}

\author{
Masataka Asada', Gun-Aajav Bayarmaa², Kengo Morohashi ${ }^{3}$, Kazuyuki Hiratsuka ${ }^{1, *}$ \\ ${ }^{1}$ Graduate School of Environment and Information Sciences, Yokohama National University, Yokohama, Kanagawa \\ 240-8501, Japan; ${ }^{2}$ Faculty of Biology, National University of Mongolia, Ulaanbaatar, Mongolia; ${ }^{3}$ Plant Biotechnology \\ Center, Ohio State University, Columbus, OH 43210, USA \\ *E-mail: hiratsk@ynu.ac.jp Tel \& Fax: +81-45-339-4413
}

Received December 19, 2006; accepted January 17, 2007 (Edited by K. Yoshida)

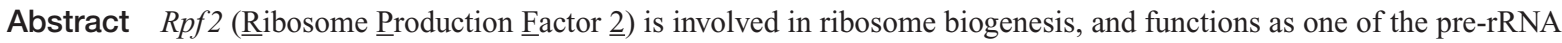
processing factors. Although properties of the Rpf2 protein in yeast have been described in detail, there have been no reports on $R p f 2$ homologues in higher eukaryotes. To investigate the function of $R p f 2$ homologues in higher plants, we identified genes encoding Rpf2-like proteins from Arabidopsis and rice and designated them AtRpf2 and $\operatorname{OsRpf2,}$ respectively. The predicted amino acid sequence revealed that both genes contain the Brix domain, a characteristic domain associated with ribosome biogenesis, and a sigma 70-like RNA binding motif, a eukaryotic RNA binding domain unique to a superfamily of proteins required for ribosome biogenesis. Both gene transcripts were detected in all organs and tissues tested and the expression patterns were consistent with the typical expression pattern of previously described ribosomal protein genes. Transient expression of GFP::AtRpf2 and GFP::OsRpf2 revealed predominant nucleolar localization within the nucleus of both fusion proteins in onion epidermal cells and tobacco BY-2 cells. These results suggest that Rpf2-like proteins in higher plants are also involved in ribosome biogenesis and function as pre-rRNA processing factors.
\end{abstract}

Key words: Nucleolar protein, ribosome biogenesis, Rpf2, rRNA processing.

Ribosomes are responsible for translating the RNA into proteins and essential for all living organisms. Eukaryotic ribosome, composed of $40 \mathrm{~S}$ and $60 \mathrm{~S}$ subunits containing four rRNAs and about 80 ribosomal proteins, is synthesized mainly in the nucleolus through many processes (Warner 1999; Woolford and Warner 1991). In Saccharomyces cerevisiae, three of four rRNAs are transcribed as a single $35 \mathrm{~S}$ pre-rRNA and are cleaved to the 25, 18, and 5.8S rRNA sequences. During rRNA processing, ribosomal proteins are transported into the nucleolus and are assembled with rRNAs to form preribosomal particles. Many of the steps in ribosome biogenesis have been studied in yeast, and most of these mechanisms are conserved throughout evolution (Kressler et al. 1999; Venema and Tollervey 1999). However, the function of each factor has not been elucidated. Previous studies have identified Rpf2 ( Ribosome Production Factor $\underline{2}$ ) as an essential gene for growth in $S$. cerevisiae. The Rpf2 protein is a pre-rRNA processing factor responsible for the processing of 27Spre-rRNA to 25SrRNA in ribosome biogenesis and interacts with Rrs1, an essential nuclear protein of 203 amino acids with an important function in the maturation of 25S rRNA in S. cereviseae (Morita et al. 2002). Rpf2 family proteins show similarity to the Imp4 protein, a specific component of the U3 snoRNP, and constitute the Imp4 superfamily in combination with Imp4, Rpf1, Brx1 and Ssf1 protein family. All of the Imp4 superfamily member proteins possess a 17-amino acid consensus sequence that constitutes a sigma-70 like RNA binding motif (Wehner and Baserga 2002).

In Arabidopsis and tobacco, previous studies indicated that disruption or down-regulation of the ribosomal protein gene is associated with various morphological abnormalities, but the mechanisms involved in phenotypic changes have not been fully clarified (Van Lijsebettens et al. 1994; Williams and Sussex 1995; Ito et al. 2000; Weijeres et al. 2001; Nishimura et al. 2005). On the other hand, there are few reports available concerning the rRNA processing factors in plants (Brown and Shaw 1998).

To investigate the functions of rRNA processing factors in plants, we conducted a series of experiments using Rpf 2 homologous genes from Arabidopsis and rice because of the availability of the genome sequence information and their versatility. Here we report some results on the database analysis, cDNA isolation and partial characterization of the Rpf2 homologues from

Abbreviations: DAPI, 4',6-Diamidino-2-phenylindole; GFP, green fluorescence protein; rRNA, ribosomal RNA; Rpf2, ribosome production factor 2; Rrs1, regulator of ribosome synthesis 1; RT-PCR, reverse transcription polymerase chain reaction This article can be found at http://www.jspcmb.jp/ 
Arabidopsis and rice.

\section{Materials and methods}

\section{Bioinformatic methods}

BLASTN and BLASTP analyses were conducted with Genome net (http://www.genome.jp/). Multiple sequence alignment analysis and construction of a phylogenetic tree were performed with the CLUSTAL W program (http://align.genome.jp/).

\section{Plant materials and growth conditions}

Seeds of Arabidopsis thaliana (ecotype Colombia) and rice (O. sativa L. ssp. japonica cv. Nipponbare) were sterilized by incubation for $10 \mathrm{~min}$ in $2 \%$ hypochlorite $/ 0.01 \%$ Tween- 20 followed by rinsing five times with sterilized water, and then germinated on MS plates. Seeds were stratified in darkness at $4^{\circ} \mathrm{C}$ for $2 \mathrm{~d}$ before being transferred to a greenhouse. Two weeks later, Arabidopsis seedlings were transferred to soil and grown in growth chambers at $23^{\circ} \mathrm{C}$ under a 12 -h-light $/ 12$-hdark cycle. Opened flowers, flower buds, stems, cauline leaves, rosette leaves, roots and siliques were harvested for RNA preparation. Two weeks after germination rice seedlings were transferred to soil and grown in an air-conditioned room under an 11-h-light, $25^{\circ} \mathrm{C} / 13$-h-dark, $18^{\circ} \mathrm{C}$ cycle. Panicles before heading, panicles after heading, leaves, culms, and roots were harvested for RNA preparation.

\section{Semi-quantitative RT-PCR analysis}

For semi-quantitative RT-PCR, total RNA from Arabidopsis and rice were isolated with TRI reagent (Sigma, product number; T9424) according to the protocol recommended by the manufacturer. We used $400 \mathrm{ng}$ of total RNA as a template for RT-PCR amplification with an RNA PCR Kit (AMV) Ver.3.0 (Takara). The cDNA fragments were amplified with specific primers AtM532-Nde5 (5'-GGGCATTATGATGGAAATACGAACTCCGAAGACT-3') and AtM532-Xho3 (5'-GGGCTCGAGTCAGAAGACACCTTCATCTTTT-3') for AtRpf2 and OsRpf2-5 long (5'-GGTCTAGAATGGTGGCGGCCATCAGGGTGCCCA-3') and OsRpf2-3 (5'-GGTCTAGATCACTCGGGATTTATCTTTTGC-3') for OsRpf2. The primer combination F-EF1 $\alpha\left(5^{\prime}\right.$-TCGAGACCACCAAGTACTACTGC-3') and R-EF1 $\alpha$ (5'-ATCATACCAGTCTCAACACGTCC-3') of the EFI $\alpha$ gene in Arabidopsis and OsActin-5 (5'-AGAGCTACGAGCTTCCTGATGGAC-3') and OsActin-3 (5'-GAGAGATGCCAAGATGGATCCTCC-3') of the Actinl gene in rice were used for RT-PCR amplification as an internal control. After 22cycles of PCR in Arabidopsis and 27cycles in rice, amplified products of internal control genes were detected by ethidium bromide staining of $1 \%$ agarose gel. PCR products of AtRpf2 and OsRpf2 were blotted onto Hybond $\mathrm{N}+$ membranes and detected with AlkPhos Direct Labeling and Detection System (Amersham) with corresponding cDNA probes.

\section{Reporter gene constructions and transient expression of the GFP fusion proteins}

The cDNA of AtRpf 2 was cloned using a $3^{\prime}$ and $5^{\prime}$ RACE system (Gibco BRL) with AtRpf2cDNA specific primers AtM532/1 (5'-GGTTGAAACTGGGAAGAAGACG-3'), MDB1
(5'-CTCTGTAATGACGGAACTCTAC-3'), MDB2 (5'-CTTTGTTCCTTCATGAGGTGC-3'), and NMDB2 (5'-CGCCCGCAAGGATTTAAAGTTC-3'), and subcloned into EcoRV site of the pT7-Blue vector (Novagen). Because of the availability of restriction enzyme sites, a cDNA fragment corresponding to AtRpf2 (69-314aa) was excised as an StuI-SalI fragment from the pT7-Blue and inserted into Xhol/blunt-Sall site of 221-EGFP-C1 vector (Bayarmaa et al. 2003). The full-length coding region of OsRpf2 was amplified with primers OsRpf25Kpn (5'-GGGGGTACCATGGTGGCGGCCATCAGGGTGCCCA-3') and OsRpf2-3Sma (5'-GGGCCCGGGTCACTCGGGATTTATCTTTTGC-3'), and the amplified fragment was digested with KpnI-SmaI. The digested fragment was subcloned into KpnI-SmaI site of 221-EGFP-C1 vector to produce the GFP::OsRpf2 fusion protein.

Onion epidermal cells and tobacco BY-2 cells were transformed transiently with $1.6 \mu \mathrm{m}$ gold particles coated with each plasmid using a model PDS-1000/He particle delivery system (Bio-Rad). The GFP localization was visualized using a fluorescent microscope, E800 (Nikon), equipped with a color CCD camera system (Hamamatsu Photonics).

\section{Results \\ Identification of Rpf2 homologues and isolation of $c D N A s$}

We identified Rpf2 homologous genes from A. thaliana (locus ID: At3g23620) and O. sativa (locus ID: R01AP002970-40060R http://cdna01.dna.affrc.go.jp/cDNA/), by a database search using the yeast Rpf2 amino acid sequence (GenBank accession number; CAA82160) as the query sequence. To investigate whether Rpf2 proteins are conserved in evolution, we searched the database and identified Rpf2 homologues from eight species. The amino acid sequence of the eight Rpf2 homologues were aligned and displayed as a phylogenetic tree showing that the gene products from plant species are clustered (Figure 1). These results suggest that Rpf2 proteins are conserved throughout evolution and that the Rpf2 homologous gene exists as a single copy in most cases including $A$. thaliana. On the other hand, sequence information for two closely related $R p f 2$-like genes were obtained from the rice genome sequence.

Because alignment of the predicted amino acid sequence revealed high homology to the sequence of $R p f 2$, especially in the sigma70-like motif region, and the Brix domain, a characteristic domain involved in ribosome biogenesis (Eisenhaber et al. 2001), we designated these two genes AtRpf2 and OsRpf2, respectively (Figure 2). The predicted amino acid sequence of these two genes from genome annotation corresponded well to that of the full-length cDNA sequence information (accession number: At3g23620: AY122939 and AY070410; R01-AP002970-40060R: AK100776, respectively). We could isolate both cDNA clones by RT-PCR using specific primers designed based 


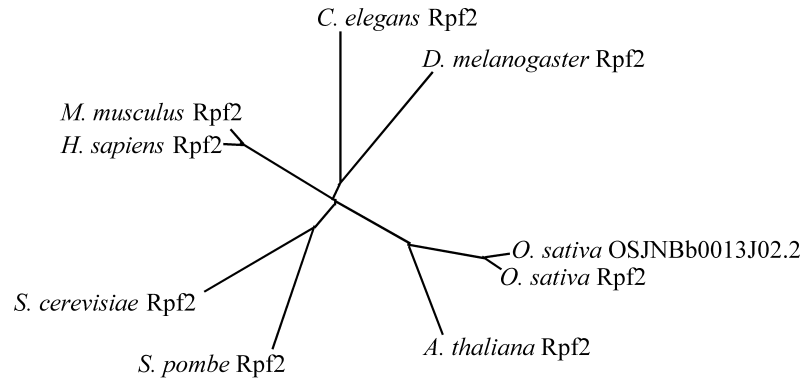

$\underline{0.1}$

Figure 1. Phylogentic tree of Rpf 2 homologues in various organisms. The distance scale represents the evolutionary distance expressed as the number of substitution per amino acid. GenBank accession number; $O$. sativa Rpf2, BAB32971; O. sativa OSJNBb0013J02.2, AAT07597; A. thaliana Rpf2, AAM67472; S. cerevisiae Rpf2, CAA82160; H. sapiens Rpf2, BAB14983; M. musculus Rpf2, BAA95117; D. melanogaster Rpf2, AAF55514; C. elegans Rpf2, AAF60765; S. pombe Rpf2, CAB54156.

on the predicted sequence of the corresponding cDNAs. The AtRpf 2 cDNA contained an ORF of 945 bp encoding a protein of 314 amino acids and the OsRpf2 cDNA contained an ORF of $933 \mathrm{bp}$ encoding a protein of 310 amino acids, respectively.

By the sequence homology search based on the Rpf2 amino acid sequence, we also identified a putative Rpf2like gene from the rice genome. The predicted gene product encoded by the OSJNBb0013J02.2 (accession number: AC129719) contains a sigma-70 like RNA binding motif, a characteristic motif of the Rpf2 family. However, from the database information, the predicted mRNA sequence of the OSJNBb0013J02.2 differed from that of the full-length cDNA information (accession number: AK106697) that is actually transcribed from the genomic region (locus ID: R05-AC129719-40020R; http://cdna01.dna.affrc.go.jp/cDNA/).

To investigate whether the mRNA sequence from OSJNBb0013J02.2 is transcribed, we attempted to obtain the cDNA derived from the predicted mRNA sequence of OSJNBb0013J02.2 (http://www.ncbi.nlm.nih.gov/ entrez/viewer.fcgi? val $=34732726 \&$ itemID $=2 \&$ view $=\mathrm{g}$ bwithparts). Amplification of the predicted cDNA by the oligonucleotide primers was confirmed by PCR using genome DNA as a template and we performed RT-PCR using total RNA samples from various organs including panicles before heading, panicles after heading, leaves, stems, and roots. However, we could not detect the amplification of corresponding cDNA fragment predicted from the OSJNBb0013J02.2. These results suggest that the Rpf2-like gene products encoded by the R05-AC129719-40020R is not expressed, if not at all, under the experimental conditions used in this study.

To obtain expression profiles, we analyzed the RNA accumulation in various samples to determine the transcription levels of AtRpf2 and OsRpf2 genes. Analysis of semi-quantitative RT-PCR demonstrated that

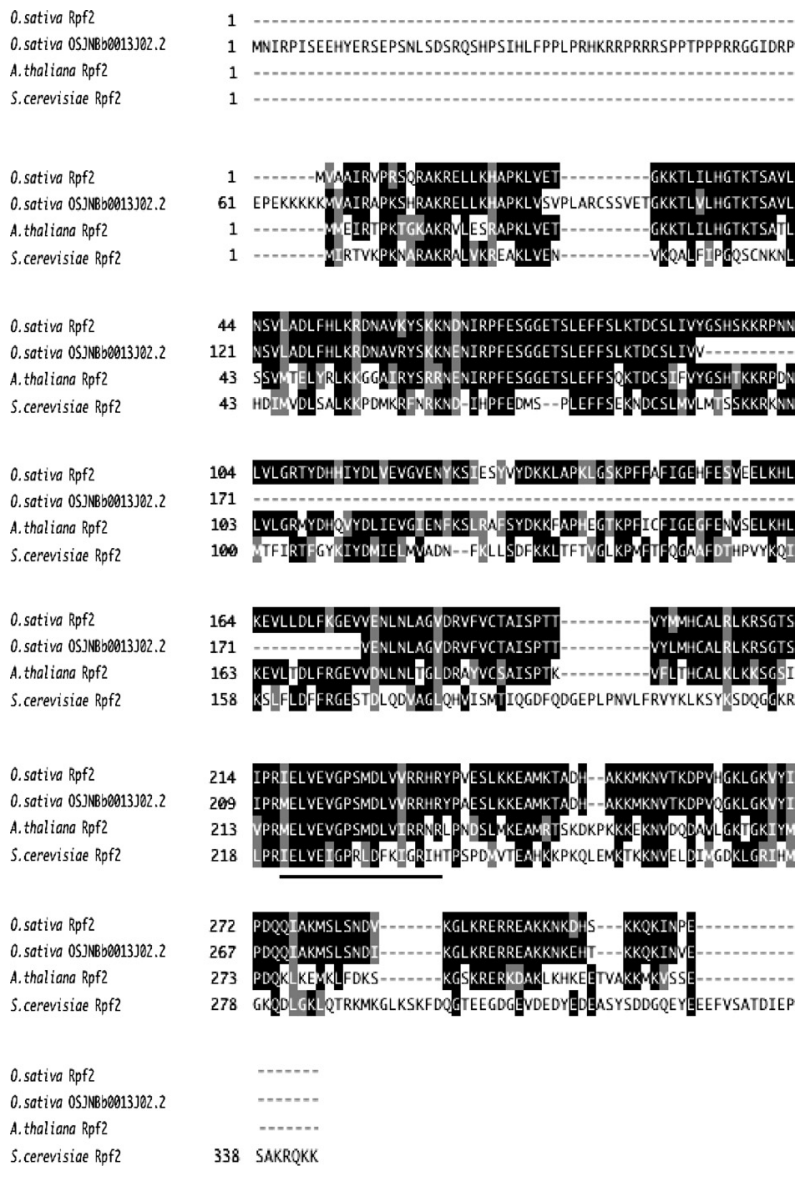

Figure 2. Multiple sequence alignment of Rpf2 homologues. White letters on black boxes indicate identical amino acids, and similar ones are indicated with shading boxes. The underlined amino acid sequences show a sigma-70 like RNA binding motif (216-233aa). GenBank accession number; O. sativa $\mathrm{Rpf2,} \mathrm{BAB32971;} \mathrm{O.} \mathrm{sativa}$ OSJNBb0013J02.2, AAT07597; A. thaliana Rpf2, AAM67472; $S$. cerevisiae Rpf2, CAA82160.

the transcripts from both genes were detected in all samples tested (Figure 3). Relatively higher expression of AtRpf 2 mRNA was observed in buds, open flowers, stems and roots of Arabidopsis. Similarly, expression levels of $O s R p f 2$ mRNA were also higher in panicles before heading, panicles after heading and leaves in rice. Although there are morphological differences between Arabidopsis and rice, the expression profiles observed in this study suggest that the expression patterns of AtRpf 2 and OsRpf 2 are essentially the same, and prominent tissue-specific expression was not observed under the conditions of this study.

\section{Subcellular localization of GFP fusion proteins}

To identify the subcellular localization of AtRpf 2 and OsRpf2 proteins, we made plasmid constructs expressing an in-frame N-terminal translational fusion with GFP. These plasmid constructs encoding GFP fused to the 69-314aa of AtRpf2 (GFP::AtRpf2) and the fulllength protein of OsRpf2 (GFP::OsRpf2), respectively, 
A

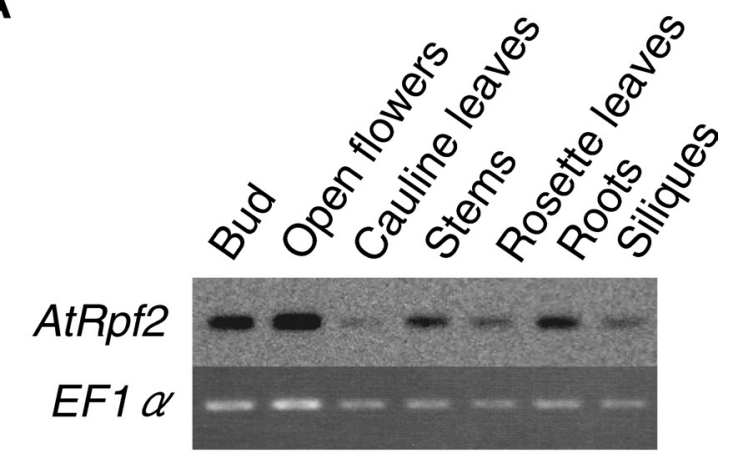

B

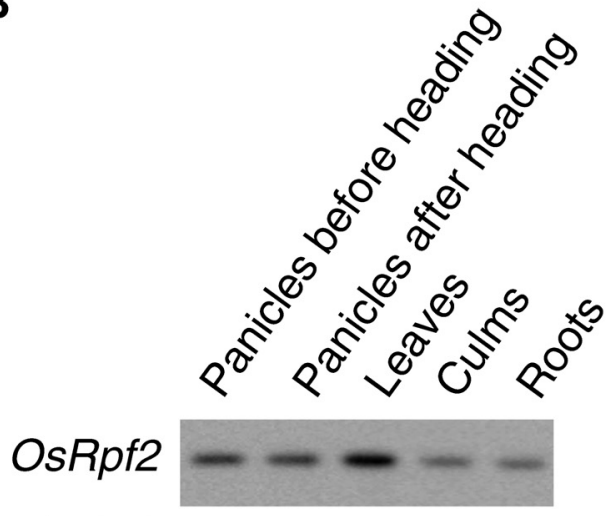

\section{Actin1}

Figure 3. Detection of mRNA expression by RT-PCR. (A) RT-PCR analysis of AtRpf 2 in various organs of Arabidopsis. RT-PCR products of the Arabidopsis EF1 $\alpha$ gene were used as internal standard. (B) RTPCR analysis of $O s R p f 2$ in various organs of rice. RT-PCR products of the rice Actin 1 gene were used as internal standard.

were introduced into plant cells by microprojectile bombardment and expressed under control of the CaMV35S promoter (Figure 4A). Plasmid constructs expressing GFP alone and GFP::GT-4 that express a GFP fusion protein were used as controls. The GT-4 is a putative transcription factor that localized to the nucleus (Murata et al. 2002). Transient expression of GFP::AtRpf2 and GFP::OsRpf2 fusion proteins in onion epidermal cells was observed by fluorescence microscopy $9 \mathrm{~h}$ after bombardment. The fluorescent signals from GFP::AtRpf2 and GFP::OsRpf2 were detected predominantly in the nucleolus and there were obvious differences in their intracellular localization compared with GFP alone. Notably, compared with GFP::GT-4 that localizes predominantly in the nucleus, nucleolar localization of GFP::AtRpf2 and GFP::OsRpf2 was evident (Figure 4B). To further confirm the nucleolar localization of both GFP fusion proteins, we conducted a transient expression assay using tobacco BY2 cells. As shown in Figure 4C, we could observe characteristic intracellular localization of GFP fusion proteins. As in onion epidermal cells, the nucleolar
A

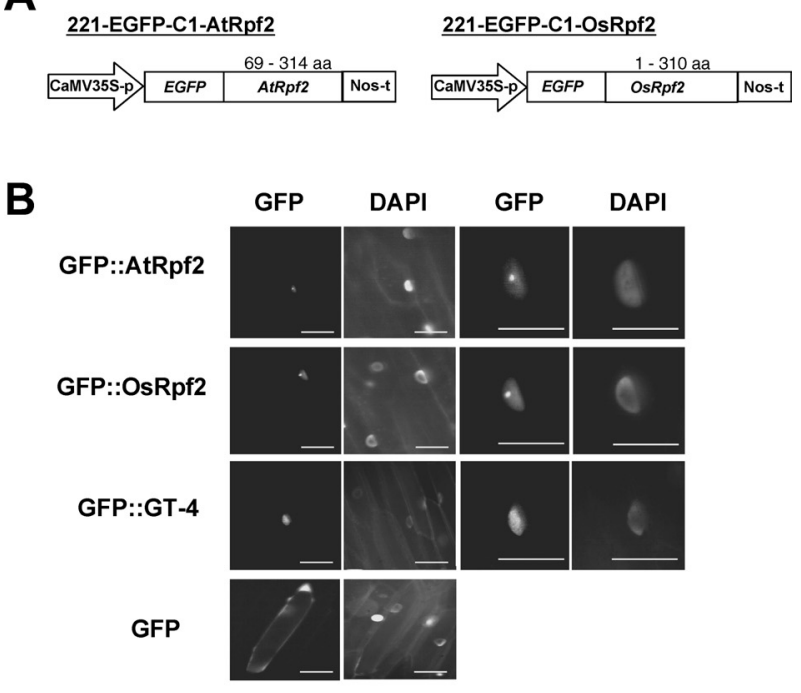

C

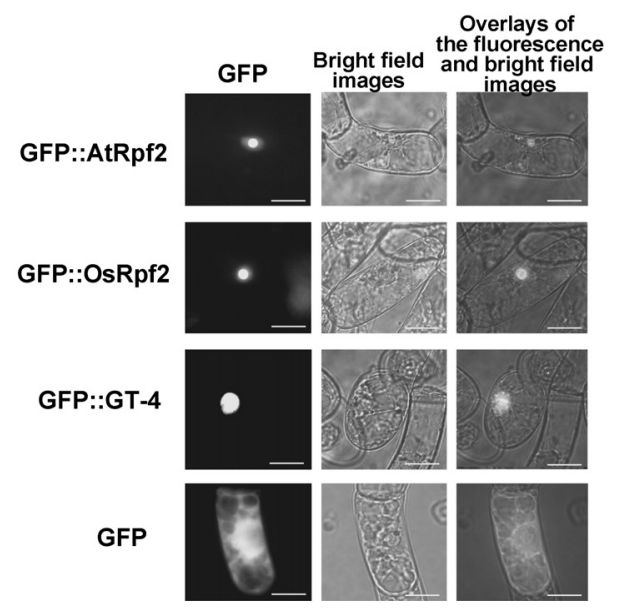

Figure 4. Subcellular localization of GFP::AtRpf2 and GFP:: OsRpf2. (A) Schematic representation of plasmid constructs for GFP::AtRpf2 (221-EGFP-C1-AtRpf2) and GFP::OsRpf2 (221-EGFPC1-OsRpf2). (B) Intranuclear localization of GFP-fusion proteins in onion epidermal cells. Epifluorescence micrographs show localizations of GFP::AtRpf2, GFP::OsRpf2, GFP::GT-4 and GFP only (left), and DAPI images (right), respectively. Bar $=100 \mu \mathrm{m}$. (C) Transient expression of GFP::AtRpf2, GFP::OsRpf2, GFP::GT-4 and GFP only in tobacco BY-2 cells. Epifluorescence micrographs on the left show GFP fluorescence alone. The central images show bright field images. Overlays of the bright field and the fluorescence images are shown in the right. $\mathrm{Bar}=10 \mu \mathrm{m}$.

localization of GFP::AtRpf2 and GFP::OsRpf2 was clearly within the nucleus in tobacco BY-2 cells.

\section{Discussion}

The $R p f 2$ was originally identified as an essential gene for vegetative growth in yeast and database analyses indicated that Rpf2 homologues are conserved throughout evolution (Wehner and Baserga 2002). In this study, we also confirmed that the Rpf2 homologues are ubiquitous across the eukaryotes. Because evolutionary conservation often indicates functional importance, we speculate that the Rpf2 genes are also essential for plant growth 
and development. In fact, our provisional results for characterization of a T-DNA insertion mutant indicate this possibility (Asada et al. unpublished data).

Although genes encoding Rpf2 homologues exist as a single copy gene in most organisms, two Rpf2 homologous sequences were found within the rice genome. High similarity between two rice homologues suggested that these genes had arisen by gene duplication. Our RT-PCR analysis indicated that only one of the two genes, R01-AP002970-40060R, is expressed. However, further investigation is necessary to confirm the expression of mRNA encoded by the other locus. Analysis of mRNA expression levels of AtRpf2 and OsRpf2 showed ubiquitous expression of both genes with some elevated expression in tissues where active protein synthesis is required. These expression profiles of both genes are similar to the typical expression pattern of ribosomal protein genes described previously (Van Lijsebettens et al. 1994; Williams and Sussex 1995; Ito et al. 2000; Weijeres et al. 2001; Nishimura et al. 2005).

In yeast, Rpf2 and its interacting protein, Rrs1, have been shown to be localized predominantly in the nucleolus (Morita et al. 2002; Wehner and Baserga 2002; Tsuno et al. 2000). Since the nucleolus is the site of ribosome biogenesis, the nucleolar localization of these proteins indicates that the $\mathrm{Rpf} 2$ and related proteins are involved in ribosome biogenesis. We investigated the intracellular localization using a transient expression assay of GFP-fusion proteins and demonstrated that the Rpf2 homologues from Arabidopsis and rice are able to direct nucleolar localization when fused to GFP. These results are consistent with a previous study on proteomic analysis of nucleolar proteins in Arabidopsis. The Arabidopsis cDNA derived from At3g23620 that encodes AtRpf2 was identified as a cDNA encoding a nucleolar protein and the GFP fusion with the protein was targeted to the nucleolus and nuclear bodies of Arabidopsis suspension cells (Pendle et al. 2005, http:// germinate. scri. ac.uk/cgi-bin/atnopdb/get-all-data?type $=$ more $\&$ value $=$ at3g23620). On the other hand, we could observe nucleolar localization of GFP::AtRpf2 and GFP::OsRpf2 proteins in onion epidermal cells and tobacco BY-2 cells, respectively. These results show that the nucleolar localization capability of AtRpf 2 and OsRpf2 is also active in heterologous species. Although the mechanisms involved in nucleolar localization of proteins are not well understood, we could observe prominent nucleolar localization of GFP-fusion proteins by transient expression assay by microprojectile bombardment. This versatile assay system is an ideal experimental approach for the dissection of nucleolar protein molecules and the identification of signals involved in nucleolar targeting of the proteins.

The findings obtained in this study suggest that the Rpf2 homologues in plants are also involved in ribosome biogenesis as a ribosomal RNA processing factor as has been reported for Rpf2 in yeast. However, further studies on biochemical as well as biological properties of AtRpf2 and OsRpf2 proteins are needed to confirm the function of Rpf2 homologues in higher plants.

\section{Acknowledgements}

This work was supported in part by Grant-in-Aid for Scientific Research (B) from Japan Society for the Promotion of Science (No. 18380197), and by the 21st Century COE Research Program "Bio-Eco Environmental Risk Management" sponsored by the Ministry of Education, Culture, Sports, Science and Technology of Japan.

\section{References}

Bayarmaa G-A, Morohashi K, Takase H, Hiratsuka K (2003) Identification of novel microsporogenesis-associated genes encoding proteins with a nuclear localization signal. Plant Biotechnol 20: 137-143

Brown JW, Shaw PJ (1998) Small nucleolar RNAs and pre-rRNA processing in plants. Plant Cell 10: 649-657

Eisenhaber F, Wechselberger C, Kreil G (2001) The Brix domain protein family - a key to the ribosomal biogenesis pathway? Trends Biochem Sci 26: 345-347

Ito T, Kim GT, Shinozaki K (2000) Disruption of an Arabidopsis cytoplasmic ribosomal protein S13-homologous gene by transposon-mediated mutagenesis causes aberrant growth and development. Plant J 22: 257-264

Kressler D, Linder P, de La Cruz J (1999) Protein trans-acting factors involved in ribosome biogenesis in Saccharomyces cerevisiae. Mol Cell Biol 19: 7897-7912

Morita D, Miyoshi K, Matsui Y, Toh-E A, Shinkawa H, Miyakawa T, Mizuta K (2002) Rpf2p, an evolutionarily conserved protein, interacts with ribosomal protein L11 and is essential for the processing of $27 \mathrm{SB}$ Pre-rRNA to $25 \mathrm{~S}$ rRNA and the $60 \mathrm{~S}$ ribosomal subunit assembly in Saccharomyces cerevisiae. $J$ Biol Chem 9: 28780-28786

Murata J, Takase H, Hiratsuka K (2002) Characterization of a novel GT-box binding protein from Arabidopsis. Plant Biotechnol 19: $103-112$

Nishimura T, Wada T, Yamamoto KT, Okada K (2005) The Arabidopsis STV1 protein, responsible for translation reinitiation, is required for auxin-mediated gynoecium patterning. Plant Cell 17: 2940-2953

Pendle AF, Clark GP, Boon R, Lewandowska D, Lam YW, Andersen J, Mann M, Lamond AI, Brown JW, Shaw PJ (2005) Proteomic analysis of the Arabidopsis nucleolus suggests novel nucleolar functions. Mol Biol Cell 16: 260-269

Tsuno A, Miyoshi K, Tsujii R, Miyakawa T, Mizuta K (2000) $R R S 1$, a conserved essential gene, encodes a novel regulatory protein required for ribosome biogenesis in Saccharomyces cerevisiae. Mol Cell Biol 20: 2066-2074

Van Lijsebettens M, Vanderhaeghen R, De Block M, Bauw G, Villarroel R, Van Montagu M (1994) An S18 ribosomal protein gene copy at the Arabidopsis PFL locus affects plant development by its specific expression in meristems. EMBO J 15: $3378-3388$

Venema J, Tollervey D (1999) Ribosome synthesis in 
Saccharomyces cerevisiae. Annu Rev Genet 33: 261-311

Warner JR (1999) The economics of ribosome biosynthesis in yeast. Trends Biochem Sci 24: 437-440

Wehner KA, Baserga SJ (2002) The sigma70-like motif: a eukaryotic RNA binding domain unique to a superfamily of proteins required for ribosome biogenesis. Mol Cell 9: 329339

Weijers D, Franke-van Dijk M, Vencken RJ, Quint A, Hooykaas P, Offringa R (2001) An Arabidopsis Minute-like phenotype caused by a semi-dominant mutation in a RIBOSOMAL PROTEIN S5 gene. Development 128: 4289-4299

Williams ME, Sussex IM (1995) Developmental regulation of ribosomal protein L16 genes in Arabidopsis thaliana. Plant $J$ 8: $65-76$

Woolford JL Jr, Warner JR (1991) In: Broach JR, Pringle JR, Jones EW (eds) The Molecular and Cellular Biology of the Yeast Saccharomyces: Genome Dynamics, Protein Synthesis, and Energetics, Cold Spring Harbor Laboratory, pp 587-626 